\title{
AN INCLUSIVE REIMAGINATION: EXPLORING ALTERNATIVE PERSPECTIVES TO AUTISM MANAGEMENT IN SOUTH ASIA
}

\author{
Vardaan Nagpal \\ Modern School Vasant Vihar \\ DOI: 10.46609/IJSSER.2020.v05i09.025 URL: https://doi.org/10.46609/IJSSER.2020.v05i09.025
}

\begin{abstract}
As research and awareness on Autism Spectrum Disorder grows in South Asia, so does the incidence of cases, from early childhood, into adulthood. The need of the hour is to develop culturally specific, individualized treatment methods for a more inclusive approach to ASD management in South Asia. Given the community based culture, often conservative cultures, and low income settings and backgrounds, developing alternative treatments which are accessible is crucial. It is further important to develop an approach which encourages viewing ASD as a different way of being and a learning approach, rather than a disability associated with a stigma. This paper will examine existing trends in autism management in the region, and shed light on promising developments in developing unique methods for the South Asian context. The paper will propose recommendations for better implementation of these methods, as well as emphasize the need for data collection and targeted interventions, which involve translation into local languages, further detailed case studies, and collaboration between countries.
\end{abstract}

Keywords: Autism, ASD, Testing, Research, Management.

\section{Introduction}

Autism is one of the world's fastest growing developmental health challenges, with up to 70 million people affected, causing a severe effect on the social development of children (PTI, 2015). There is currently a gap in the knowledge about the incidence of ASD in South East Asian countries, due to lack of awareness and testing. Lack of significant authorship and research output for low and middle income countries (LMICs) stunts the development of evidence-based health policies and practice in LMICs (Hossain, 2017). While numbers are on the rise across South Asia, this is likely due to more awareness and testing rather than a greater prevalence of autism (Thomas, 2019). Admittedly, the number of individuals living with autism could be much higher in South Asia - especially in rural areas where it may not be reported or misinformation about the condition still exists (Thomas, 2019). 


\section{International Journal of Social Science and Economic Research}

ISSN: $2455-8834$

Volume:05, Issue:09 "September 2020"

There continues to exist a considerable level of misinformation regarding ASD in South Asian countries, leading to incorrect diagnosis and viewing it as one of the folk illnesses. Due to the particular conditions in these countries, there is a great need for cultural considerations of mental illness and ASD. Taking these cultural factors into account, developing alternative, accessible and cost-effective practices for ASD management need to be developed. Doctors in these countries often have minimal experience with autism and so may fail to recognize its features (Divan, 2017). When a child does receive a diagnosis, South Asian families still have to find appropriate treatments in centers with suitable therapists, which are typically located in large cities (Divan, 2017). The families must travel, often long distances, for regular appointments, and this adds to their strain (Divan, 2017).

This paper will shed a light on the existing gaps in knowledge and implementation of management techniques in South Asian countries, examining current psychological theories and methods of intervention. The paper will provide a review of alternative treatment methods which are proving effective, and how existing methods could be adapted to the particular cultural contexts of countries like India. The paper will conclude with policy recommendations, emphasizing on the dire need for culturally sensitive case studies, trials, and development of treatment methods, aiming to foster a more inclusive approach rather than a stigmatization of ASD as a disability.

\section{Background}

There has been an encouraging increase in awareness regarding Autism Spectrum Disorder, or ASD, in both children, adolescents and adults. In general, relatively little has been written on autism in developing countries in South Asia as compared with what has been published on autism in North America and Europe (Barua and Daley, n.d). From the late 1980s through today, autism related research, treatment and therapy in South Asia has experienced an intense period of activity relative to the previous decades (Barua and Daley, n.d). Growth has occurred in numerous domains: diagnosis, treatment and educational options, parental involvement, involvement of civil society organisations, vocational options, human resource development, and legislation (Barua and Daley, n.d). On a regional-level, awareness about autism has risen over the years - especially with mass media playing a key role in disseminating information and breaking long-held stereotypes (Thomas, 2019). Various initiatives have been implemented and many organisations have been formed across Southeast Asia to give the condition attention and increase support for autism programmes (Thomas, 2019).

Once considered rare in South Asia, the current understanding is that autism is in fact one of the more common developmental disabilities (Juneja and Sairam, 2018). The increase in prevalence can be attributed mainly to increased awareness amongst professionals. Changes in case 


\section{International Journal of Social Science and Economic Research}

ISSN: $2455-8834$

Volume:05, Issue:09 "September 2020"

definition, earlier detection, and diagnostic substitution of cases may also be contributory (Juneja and Sairam, 2018). It is crucial to note that symptom expression and initial diagnosis that leads to early intervention for children is also heavily influenced by socio economic and cultural contexts.

In lower income and more conservative settings in India and other South Asian cultures, pharmacological intervention remains the norm - these could include methods such as acupuncture, acupressure, Auditory Integrated Therapy, traditional or indigenous medicine, behavior therapy, magneto therapy, Dimethylglycine facilitated communication, etc (Barua and Daley, n.d). 'Expertise' of astrologers, faith healers, Fakirs, family counseling, family guru, and such others are also sought (Barua and Daley, n.d). Given that these methods are not based on any scientific evidence or proof of improvement of ASD in children, these should be banned by the government through public awareness programs.

In the South Asian context, there is still considerable stigma and discrimination affecting children with autism and their families (Minhas et al, 2014). Specialist services are rare, concentrated in urban areas, and inaccessible to the majority (Minhas et al, 2014). Strategies for intervention should include building community and family support networks to provide respite to the main caregiver (Minhas et al, 2014). In the absence of specialists, community members such as community health workers, traditional practitioners and even motivated family members could be trained in recognizing and providing evidence-based interventions (Minhas et al, 2014). Such task-shifting strategies should be accompanied by campaigns to raise awareness so greater inclusivity can be achieved (Minhas et al, 2014).

What is crucial especially in the context of these countries is developing an understanding of autism not as "disorder" but of an alternative way of being, to prevent discrimination as well as to avoid the stigma of families believing it is a liability. The following section will elaborate on this developing understanding, and alternative modes of intervention which are proving effective.

\section{Discussion}

In South Asian countries like India, available standard forms of therapy are mostly Applied Behavioural Analysis (ABA)-based and include an eclectic mix of play-based interventions, relationship development interventions, sensory integration, social skills training, special education, speech and language therapy and group therapy (Juneja and Sairam, 2018). However, the therapies are usually not as intensive or clearly defined as recommended in Western literature (Juneja and Sairam, 2018). With increasing awareness of the need for cultural context to be taken into account, there has been an increased use of teaching interventions, which are more cost 


\section{International Journal of Social Science and Economic Research}

ISSN: $2455-8834$

Volume:05, Issue:09 "September 2020"

effective, and can be adapted to be implemented in the household, involving the parents. This has proven particularly effective in the South Asian context, and more effective than pharmacological treatments (Sharma and Rangarajan, 2019). Studies have found that the use of an eclectic approach in making choices regarding teaching strategies, incorporating varied research based effective teaching practices, and following a systematic and informed approach of assessments - teaching interventions - ongoing evaluation are key to supporting students with ASD (Sharma and Rangarajan, 2019).

The PACT and PASS treatments incorporating play therapy have proved to be useful in the South Asian context, and have been able to incorporate technological aids for autism treatment and caregiver support, at the stage of child development. Early intervention can prove to be vital for the future development into adult hood and managing ASD. Due to the cost effectiveness of parent centered play therapy, it has found promising results in the context of South Asian countries which are firstly lower income environments, and secondly, are cultures centered around the family structure and parental involvement more so than western cultures. In this respect, the Preschool Autism Communication Therapy (PACT) has been a radical approach for developing countries such as India, because it involves training non-specialists to support and guide parents, allowing children and families with few resources to receive the care they need and deserve (Divan, 2017). This is also one of the first initiatives in the world that aims for a more culturally nuanced therapeutic method which incorporates elements of play therapy (Divan, 2017).

In essence, the adapted intervention is based on the same theoretical construct as PACT and utilises a naturalistic approach to scaffolding and developing communication skills in the child with ASD (Divan et al, 2015). The findings accounted for adaptation in some aspects of its content and delivery to enhance contextual acceptability and to enable the intervention to be delivered by non-specialists (Divan et al, 2015; Divan, 2017). In the cultural context of grandparents or nannies being the primary caregivers, flexibility in the adult targeted for the intervention was introduced. Despite concerns of experts about the 'reflective' nature of the intervention, parents were able to adapt to this approach with ease during video-feedback (Divan et al, 2015). This revolutionary treatment for behavioural management of ASD has paved the way for a future of individualized treatment planning for ASD in the Indian context, and the South Asian context. The focus of all stakeholders must be individualized treatment planning, and developing laws and policies to promote and protect the rights and empowerment of persons with autism (Thomas, 2015). 


\section{International Journal of Social Science and Economic Research}

ISSN: $2455-8834$

Volume:05, Issue:09 "September 2020"

\section{Conclusion}

While increased awareness and progressive treatments are surely a step in the right direction, it is also important to analyze whether the methods of treatment and inclusiveness of policy has improved in a substantial manner alongside the same. In order to do so, researchers must take into account income levels, socio economic contexts, and cultural contexts in order to adapt treatment methods to most benefit those with ASD in varying situations. This underscores the importance of an integrated, culturally sensitive approach to ASD management. First and foremost, it is important to acknowledge that only a limited number of studies on ASD have been conducted in South Asia that limit any conclusion despite the alarming increase in the prevalence of ASD in recent years (Hossain, 2017).

To know the extent of ASD as a public health problem, there is an urgent need for all countries of this region to conduct well-designed epidemiological research using uniform and appropriate tools (Hossain, 2017). Knowing the prevalence could help choosing screening and diagnostic tools that are applicable, culturally acceptable, and cost-effective to identify individuals who can benefit the most from early diagnosis and intervention, which is key to maximizing the impact of effective treatment on improving long term prognosis for autism in India, and other countries in the region (Hossain, 2017). It will be crucial for countries in the region to create, expand and improve health information and surveillance systems to gather appropriate sex- and agedisaggregated data, as well as other relevant information on ASD; collate and routinely report findings (WHO, 2017).

Creating a support system is crucial in the community based South Asian culture. Countries must ensure services and support for families and caregivers through the health and social services sector by provision of social support, respite care and promotion of caregiver support groups (WHO, 2017). They must also establish greater collaboration among "informal" health-care providers, traditional or indigenous practitioners, as well as religious leaders, school teachers, etc. and nongovernmental and caregiver organizations (WHO, 2017). Ensuring an appropriate number and equitable distribution of competent, sensitive and appropriately skilled professionals and workers who can offer culturally appropriate services for persons with ASD is important, as well as improving the capacity of care workers in the field to promote early detection and management of ASD, and deliver evidence-based psychosocial interventions and referral as appropriate to other levels of care and services (WHO, 2017).

In order to foster an inclusive approach which treats autism as a learning style rather than a burdensome disability, countries in this region must establish a rights-based approach for helping those with ASD and their caregivers, which should include appropriate institutional, legal, financial and service arrangements (human resource, technological, logistic), including an 
International Journal of Social Science and Economic Research

ISSN: 2455-8834

Volume:05, Issue:09 "September 2020"

institutional focal point (WHO, 2017). The end-goal for therapeutic methods relating to ASD in children must be educational justice and a change in societal attitudes (Merry, 2019; Barua and Daley, n.d). PACT is useful in this respect, especially in India, where parents are not expected to have an opinion in the kind of interventions that the child is put on (Barua and Daley, n.d).

By involving parents and family members in the treatment, a more positive familial environment is created, thus improving the status of the child as a child whose needs must be accommodated for, rather to be seen as a liability to the family. Giving parents skills that allow them to generalize strategies across multiple areas of the child's life (Divan, 2017). It is an alternative and more inclusive approach to equip children with the skills they need to be as independent as possible, and function effectively in their environment to the greatest extent possible (Merry, 2019). Such an approach will ensure that not only is there protection and care of children with autism, but also an increase in the child's active participation and independent functioning in their environment, which benefits them as they grow (Merry, 2019).

\section{References}

Barua, M, and Daley, T, (n.d), 'Autism', Rehabilitation Council of India, http://www.rehabcouncil.nic.in/writereaddata/autism.pdf

Divan, G, (9 May 2017), 'Novel autism treatment translates well to South Asian nations', Spectrum, https://www.spectrumnews.org/opinion/viewpoint/novel-autism-treatment-translateswell-south-asian-nations/

Divan, G, et al, (2015), 'Adapting an evidence-based intervention for autism spectrum disorder for scaling up in resource-constrained settings: the development of the PASS intervention in South Asia', Global Health Action, Vol.8, Issue 10

Hossain, M, et al, (2017), 'Autism Spectrum disorders (ASD) in South Asia: a systematic review', BMC Psychiatry, Vol.17, Issue 281

Juneja, M, and Sairam, S, (15 March 2018), 'Autism Spectrum Disorder - An Indian

Perspective', Recent Advances in Autism, SM Group,

http://www.smgebooks.com/autism/chapters/AUT-18-12.pdf

Merry, M, (2019), 'Do Inclusion Policies Deliver Educational Justice for Children with Autism? An Ethical Analysis', Journal of School Choice, Vol 14, Issue 1 
Minhas, A, et al, (2014), 'Parents' perspectives on care of children with autistic spectrum disorder in South Asia - Views from Pakistan and India', International Review of Psychiatry, Vol.27, Issue 3

PTI, (17 December 2015), 'New autism treatment to help children in India', The Economic Times, https://economictimes.indiatimes.com/news/science/new-autism-treatment-to-helpchildren-in-india/articleshow/50218946.cms

Sharma, U and Rangarajan, R, (2019), 'Teaching students with autism spectrum disorders in South Asia: a scoping study and recommendations for future', International Journal of Developmental Disabilities, Volume 65, Issue 5

Thomas, J, (5 March 2019), 'What is South East Asia doing about autism?', The Asean Post, https://theaseanpost.com/article/what-southeast-asia-doing-about-autism

WHO, (2017), 'WHO South-East Asia Regional Strategy on Autism Spectrum Disorders', https://apps.who.int/iris/bitstream/handle/10665/259505/9789290225454en.pdf? sequence $=1 \&$ is Allowed $=y$ 